which enabled this work to be done. We are also indebted to Dr. R. J. Lythgoe for the photographs.

\title{
REFERENCES
}

Bourne, M. C., Campbell, D. A., and Tansley, K.-Hereditary degeneration of the rat retina. Brit. Jl. Ophthal. (in press), 1938.

Calmoun, F. P.-Bilateral coloboma of the optic nerve. Arch. of Ophthal., Vol. III, p. 71, 1930.

Conts, G. - The pathology of coloboma at the nerve entrance. Roy. Lond. Ophthal. Hosp. Rep., Vol. XVII, p. 178, 1908.

Ginsberg, S.-Ueber die angeborenen Coloboma des Augapfels. Centrabl. $f$. prakt. Augenheilk., Bd. XX, p. 225, 1896.

Goerlitz, M.-Anatomische Untersuchung eines sogenannten Coloboma Nervi optici. Arch. f. Augenheilk, Bd. XXXV, p. 219, 1897.

KaYANaGI, Y.-Embryologische Untersuchungen ueber die Genese der Augenkoloboma und des Mikrophthalmus mit orbitalcyste. Arch.f Ophthal., Bd. CIV, p. 1, 1921.

Parsons, J. H., and CoATs, G.-A case of orbital encephalocele with unique malformation of the brain and eye. Brain, Vol. XXIX, p. 209, 1906.

van Duyse, G. M.-Colobomatous and Microphthalmic eyes. Brit. Jl. Ophthal., Vol. III, p. 529, 1919.

vON HIPPEL, E.-Graefe-Saemisch Handbuch des gesamt. Augenheilk., Pt. I, Vol. II, chap. IX, p. 27, 1900.

\section{THE APPARATUS OF PROFESSOR ARGAÑARAZ FOR EXTRACTION OF CATARACT BY THE SUCTION METHOD (PHAKOERISIS)}

THE apparatus designed by Professor Argañaraz for the operation of phakoerisis consists essentially of an ordinary glass 20 c.c. syringe (B) with its plunger (A), together with a rubber tube having moderately resistant walls (C), capable of occlusion or release by a clamp (D) and finally, the small suction cup or erisiphake (E). See Fig. 1.

The Syringe.-This is a glass syringe of 20 c.c. capacity which is capable of producing a vacuum of 5,10 or 15 c.c., representing a negative pressure of 30,50 or $70 \mathrm{~mm}$. $\mathrm{Hg}$. This vacuum can be regulated at will. If the clamp is closed and the piston withdrawn as far as the zero mark a vacuum of 15 c.c. is produced which is the longest possible with this size reservoir (A). If the plunger is only withdrawn as far as 3,5 or 10 c.c. corresponding intensities of negative pressure can be obtained.

In order to ensure that the system is hermetically sealed, it is essential to introduce a fair amount of sterilised lanoline or other suitable fat around the piston to prevent the infiltration of any air. Metal syringes are more costly and difficult to make than glass ones. They also change their shape slightly with repeated sterilisation and are harder to replace.

The retention clip (A).- This allows the piston to be fixed in 


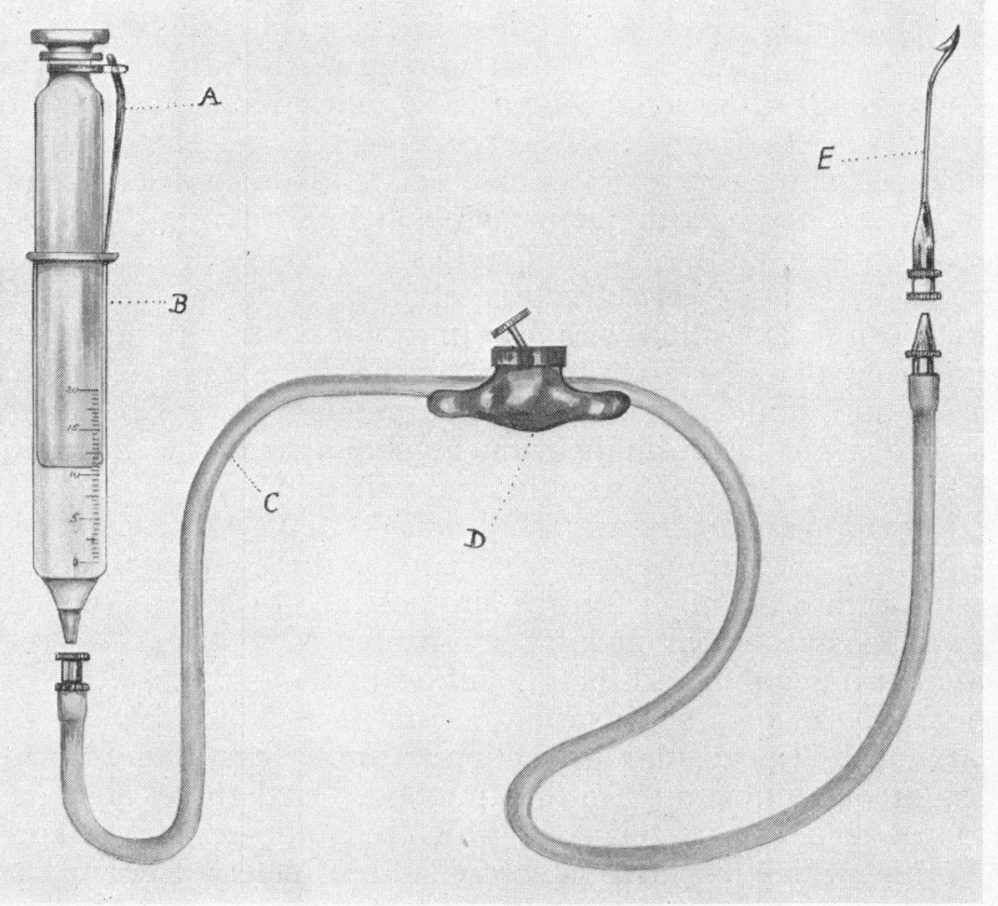

FIG. 1.

position so as to maintain the vacuum which will remain for a long time if the syringe has been smeared with lanoline. The clip (A) is fixed to a metal band around the neck of the piston and is held to this by a small screw. If the syringe breaks all that is necessary is to release the screw and the whole clip can be easily fixed to a fresh syringe.

The rubber tube.-This has a bore of $0.4 \mathrm{~cm}$. and the walls are sufficiently rigid to withstand the vacuum. The tube can be boiled many times without spoiling it.

The clamp (D), which must be controlled by an assistant, is similar to those used in blood transfusions. It is only necessary to press the button of the clamp gently between the thumb and the first finger to establish direct communication between the vacuum in the syringe and the suction cup of the erisiphake. Obviously the clamp must be closed, i.e., compressing the rubber tube, when the vacuum is produced in the syringe. The syringe and clamp are in the hands of the assistant during the operation.

The suction cup or erisiphake.-The cup or true pneumatic forceps fits into the rubber tube by a bulbous end. The small 
spoon-like extremity is $2 \mathrm{~mm}$. deep and $4-5 \mathrm{~mm}$. in diameter. Two types have been constructed, in stainless metal and in silver, one rounded with a diameter of 4.5-5 mm., and the other oval similar to that used by Barraquer which is $3.5 \mathrm{~mm}$. broad by $5 \mathrm{~mm}$. long.

Operative Technique.-The erisiphake is not an instrument which automatically removes cataracts and one must necessarily familiarise oneself with its principle before adopting it. When one has overcome the first difficulties and has used it on 15 or 20 cases it certainly gives a greater feeling of confidence and security than does the operation with Elschnig's forceps. It must always be realised that the erisiphake is at the same time a pneumatic forceps and zonuletome. Those surgeons who are not familiar with the use of such an instrument should read the instructions below in order to avoid trouble and disillusionment.

Disadvantages of Elschnig's forceps and advantages of the erisiphake.-When one has tried two or three times to seize the capsule with Elschnig's forceps and has failed, the lens practically always becomes spontaneously subluxated so that loss of vitreous is: almost inevitable and the difficulties of the extraction are much increased. If such subluxation occurs there is nothing for it but to attempt extracapsular extraction, using a cystitome, or intracapsular extraction by Smith's method. All these manoeuvres tend towards loss of vitreous which complicates or vitiates an operation, which might have succeeded completely if begun by the older methods. Many young surgeons, aware by experience of these dangers prefer to operate by the old extracapsular method which gives the greatest security and the least risk. Even in the hands of experienced surgeons the number of successful intracapsular extractions does not exceed 60 per cent. It is possible that this percentage might be improved by Arruga's technique of checking the condition of the forceps by the slit-lamp before operating. But it is an indisputable fact that when the forceps fail, which often happens, the lens is dislocated, there is loss of vitreous, cystotomy becomes difficult and in a word the use of the forceps has vitiated an extracapsular operation which might have been very satisfactory. By using a good suction apparatus all these inconveniences disappear since though it still may be difficult to luxate the lens, and the cup may slip off, if this happens the apparatus can be reapplied without difficulty or danger. The application of the Argañaraz erisiphake is made by holding the suction cup attached to the flexible rubber tube between the thumb and index finger and placing it gently on the anterior surface of the lens at the centre or anterior pole. On the other hand we should remember that the gripping of the lens by Elschnig's forceps is made completely in the dark since one cannot see the end of the forceps the surgeon does not know whether he has 
succeeded in catching the lens until this is already appearing and the zonule ruptured, when the iris is pushed forwards at the moment of delivery through the pupil. In this respect the present apparatus has the big advantage that it is possible to see and control directly the point of application of the suction cup.

If the pupil has contracted during the cutting of the corneal flap it is easy to introduce the cup through the pupil and by small lateral movements to ensure that the pupillary margin is not caught by it. Thus one can avoid injuring the iris with the edge of the cup when making the vacuum. The clamp and the vacuum syringe are worked by the assistant and the surgeon need not worry about them. When he has applied the erisiphake in this way he asks the assistant to loosen the clamp to establish communication with the vacuum in the syringe so that the lens is held firmly. 'This is the most delicate moment of the operation since now the suspensory ligament has to be ruptured and this in young patients, under 40 years with immature cataracts may offer strong resistance.

To rupture the zonule before extraction the following procedure is to be adopted :-

1. Without exerting any pressure on the eye and by means of the suction cup the lens should be partially rotated on an anteroposterior axis, the fingers moving the applicator from one side to the other of the corneal wound. This ruptures many of the zonule fibres. 2. To complete the rupture of the remaining zonule fibres one imparts to the lens, without exerting any pressure on it, lateral " rolling " and antero-posterior " pitching " movements. In this way all the fibres of the zonule are ruptured.

3. To free the lens from all adhesions before removal it should be turned through an angle of $180^{\circ}$ by means of the suction cup so that its posterior surface is in contact with the cornea. By continuing this movement it is extracted, forwards and upwards, that is to say through the corneal wound. In old subjects with mature cataracts the zonule is very weak and the lens can be removed from the eye without the manoeuvres mentioned above for rupturing the zonule. The younger the patient and the less mature the cataract the greater may be the resistance of the zonule and during the process of rupturing it the applicator may spring off the lens. This is not of much importance as one can take a second or even a third hold, recharging the syringe as often as is necessary to rupture the zonule and complete the extraction.

In these cases the traction on the zonule may produce a slight ciliary inflammation which shows itself as post-operative pain and delayed formation of the anterior chamber.

These symptoms, which can also follow the use of forceps are not of much importance and can be advantageously treated with fomentations and atropine. 
In more than 40 patients on whom we have operated with this apparatus there has been no failure to extract the lens in toto.

The syringe can be charged (or rather emptied to produce the vacuum) by the assistant immediately after the surgeon has performed the section and the iridectomy, since if it is prepared at the beginning of the operation a little air may filter in and spoil the vacuum while the surgeon is performing the preliminary steps.

\section{Iridectomy}

An iridectomy should always be performed since one never knows whether the iris will prolapse the next day. Iridectomy ensures that no incarceration will occur. One can perform Hess's peripheral iridectomy or better that of Elschnig. For this the iris must be caught at the periphery and a very fine iris forceps as well as a very small de Wecker's scissors are necessary. This is followed by a reduction or smoothing out of the upper part of the pupil by an iris repositor or the blade of the de Wecker's scissors. If the mydriasis is very marked, such as is produced by subconjunctival injection of adrenalin, it is not easy to perform Hess's iridectomy. Sometimes it is almost impossible to pick up the iris in the periphery. In these cases a total iridectomy dividing the sphincter is preferable to no iridectomy at all on account of the danger of incarceration. An excellent mydriasis is usually obtained by hourly instillations of three or four drops of 2 per cent. homatropine for four or five hours before the operation. Maximum mydriasis renders the extraction easier. A drop of 1 in 1,000 adrenalin solution injected subconjunctivally near the limbus an hour before the operation gives a maximum mydriasis. Any contraction of the sphincter seriously hinders the passage of the lens through the pupil. A good mydriasis can also be obtained with glaucosan, Arruga.

The turning back of the corneal flap and the "open operation" of extraction has the disadvantage of producing a traumatic keratitis which shows itself as an opacity of the upper part of the cornea which takes several days to disappear. The corneal flap should be turned back as little as possible when doing the iridectomy. If one is doing a total iridectomy it is better to draw the iris out through the wound. The use of this simple form of erisiphake constitutes in our opinion a further improvement comparable to that marked by the introduction of Elschnig's forceps. We have no doubt that our erisiphake will supersede the forceps in the near future. Finally to obtain the best results we recommend that no departure be made from the general modern operative technique, that is to say- 
1. Operate with the patient in bed using local anaesthesia (5 drops of 3 per cent. cocaine).

2. Facial akinesia, using a parotid injection of Bayer novocaine-adrenalin 5 per cent.

3. Mydriasis by homatropine ( 2 per cent.) 3 or 4 drops every hour, four hours before the operation.

4. Retro-ocular injection of 1 c.c. novocaine-adrenalin 1 per cent.

5. Fixation of the eye by Elschnig's suture under the superior rectus or through the episcleral tissue, as well as using the speculum, to raise the upper lid without exerting pressure.

6. Cutting a corneo-conjunctival flap and inserting a suture in this.

7. The peripheral or complete iridectomy according to degree of mydriasis taking care not to evert the corneal flap more than is necessary.

8. Without exerting any pressure above the eye apply the cup of the erisiphake smoothly to the anterior face of the lens, instructing the assistant in the meantime to release the clamp which produces the vacuum.

9. Rupture of the zonule. It is indispensable before extracting the lens to rupture the zonule by means of three movements, viz. : 1. Rotary, turning the lens on its own antero-posterior axis, displacing the handle of the erisiphake from one extremity of the wound to the other. 2. Imparting lateral rolling movements to the lens, and 3, antero-posterior pitching movements.

10. By freeing the lens from the zonule it can be extracted direct or better it can be rotated through $180^{\circ}$ so that the posterior surface comes in contact with the cornea and is drawn out through the wound.

11. The conjunctival suture is tied and another inserted in the skin of the lid to keep the eye closed on account of the akinesia.

The Electric Vacuum Pump and the inconveniences of managing the clamp on trigger of the vacuum when this is fixed to the handle of the erisiphake

The electric motor generator invented by Barraquer to produce the vacuum required for the operation has been one of the drawbacks of the method and has prevented it becoming general. The small electric motor with its charge of oil requires constant care to ensure the satisfactory working of all the parts. Moreover the key or trigger interposed between the cup and the handle which the surgeon must release to produce communication with the vacuum, necessitates the performance of an awkward manoeuvre which makes it difficult to keep the cup gently in contact with the surface of the lens. If the two surfaces are not properly in 
contact the suction of the electric motor may prove of great danger to the eye.

It is difficult for the surgeon to keep his hand steady while engaging the lens, since he must at the same time exert pressure with his finger on the trigger which puts him into communication with the vacuum. The same inconvenience is seen in the syringe vacuum apparatus of Arruga and Lijo Paoia.

Further the capacity of the syringe in this instrument was only 5 c.c., which does not seem to produce a strong enough vacuum and the frequency with which the lens slips off the cup can be put down to the feeble suction. We have seen Arruga recharge the syringe several times during the same operation.

For this reason we have adopted a syringe of 20 c.c. which can produce different degrees of vacuum between 5 and 20 c.c.

With a mature cataract and a feeble zonule in a patient of 60 , 5 c.c. of vacuum is enough to extract the lens. This is equivalent to a suction of $30 \mathrm{~mm}$. $\mathrm{Hg}$ while 15 c.c. equals $70 \mathrm{~mm}$. $\mathrm{Hg}$.

In certain patients, particularly those under 50 and with unripe cataracts the zonule is more resistant and a 10 c.c. vacuum is required to luxate the lens without having to reapply the cup several times while rupturing the zonule. 10 c.c. of vacuum is enough to remove the lens in most cases.

\section{WHITE OAK HOSPITAL, 1933-37}

\section{The following report on White Oak Hospital has been submitted by Mr. Arnold Sorsby, visiting Ophthalmologist to the Hospital}

The considerable administrative changes that have occurred at White Oak Hospital during the past two-and-a-half years make a comparative review of the work of the past five years desirable. The subjoined table gives the admissions for 1933-37, set out in the main groups of affections treated.

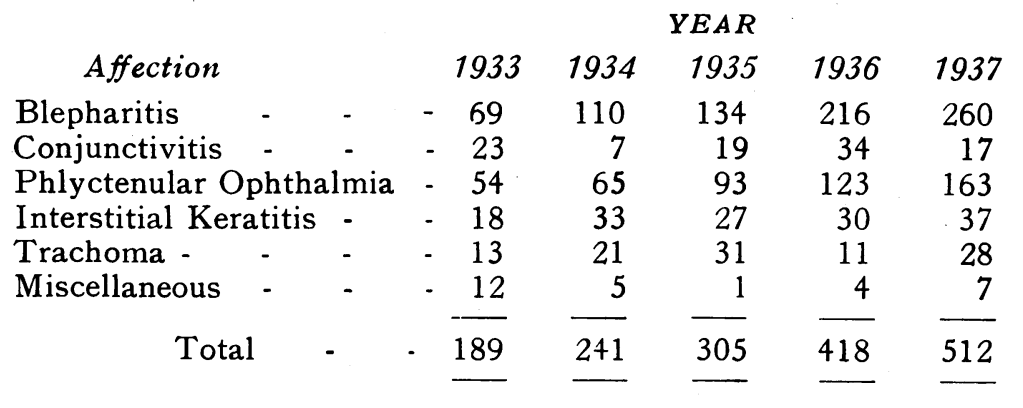

